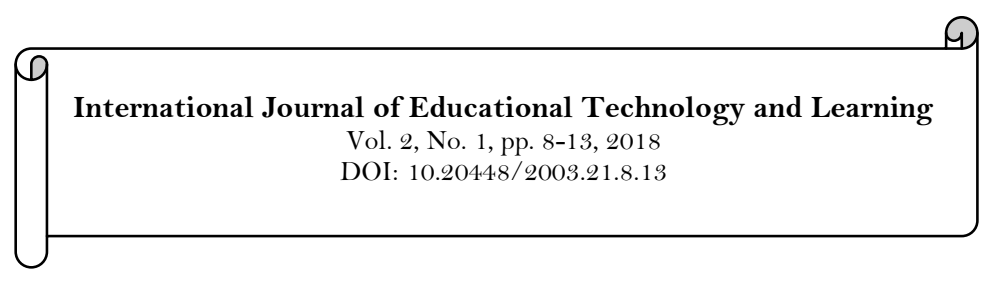

\title{
Engineering Mathematics and Modern Technology
}

\author{
Qefsere Doko Gjonbalaj ${ }^{1}$ \\ Luigj Gjoka ${ }^{2}$
}

${ }^{1}$ Faculty of Electrical and Computer Engineering-Department of Math; University of Prishtina "Hasan Prishtina", Prishtinë, Kosova.

Email: qefsere.gjonbalaj@uni-pr.edu

${ }^{2}$ Department of Engineering Mathematics, Polytechnic University of Tirana, Albania.

\begin{tabular}{|c|c|}
\hline Abstract & \\
\hline $\begin{array}{l}\text { Technology has become an essential tool for doing mathematics in } \\
\text { today's world. It can be used in a variety of ways to improve and } \\
\text { enhance the teaching of mathematics. Technology can provide } \\
\text { students with opportunities to explore different representations of } \\
\text { mathematical ideas and support them in making connections both } \\
\text { within and outside of mathematics. The use of technology in } \\
\text { teaching mathematics activities may be regarded as a new language } \\
\text { of communication in the development of construction of knowledge. } \\
\text { Knowledge about the role of technology in the educational process } \\
\text { can contribute to better preparation of future teachers in not only } \\
\text { choosing the right technology but also the way of teaching. Our } \\
\text { main goal, of all efforts in this regard, is the connection between } \\
\text { mathematics and technology. }\end{array}$ & $\begin{array}{l}\text { Keywords: } \\
\text { Modern technology } \\
\text { Engineering mathematics } \\
\text { teaching. } \\
\text { Technology and mathematical } \\
\text { education. } \\
\text { Licensed: } \\
\text { This work is licensed under a } \\
\text { Creative Commons Attribution } \\
\text { 4.0 License. } \\
\text { Publisher: } \\
\text { Scientific Publishing Institute }\end{array}$ \\
\hline
\end{tabular}

\section{Introduction}

Technology can be used in a variety of ways to improve and enhance the teaching and learning of mathematics. It can be used to facilitate mathematical discovery, understanding, and connections that may be difficult or impossible. The advantages of the technology as teaching aid is the possibility of more realistic modelling in problem solving and interdisciplinary activities, so new and reformulated disciplines in the curriculum of teacher preparing courses come up.

In the science of mathematics, analytical methods and numerical computational methods have been developed in parallel throughout the history of its development. The need to perform mathematical immediately and quick calculations, imposed by military technology (ballistics and decoding), the exploration of universe etc., has been a strong incentive for the development industry of the electronics in general and computers in particular. Construction of high-speed computers helps mathematicians to calculate and make the situation more clearly than ever before.

The meaning of calculation would not make sense without mathematics, and what led to the creation of the concept of the computer programming were the mathematical analysis methods from mathematicians, philosophers and engineers.

Indeed, two mathematicians Von Neumann in the US and Turing in England, are known as the founders of modern computers. For the analysis of computer calculations and attempts that make it as reliable as possible, we need the advanced mathematics, and this need is growing. Until the computer is programmed, it is just a box made of metal, glass, silicone etc. The program represents algorithms in the appropriate forms for the computer. Mathematics is necessary as a language for detailed description, how should it be done and when should it be done, and verifying that the program and algorithm are working correctly.

The role of mathematics, advanced mathematics in particular, is crucial to the development of modern technology. Even non-mathematical knowledge, which enables the development of technology, would not be possible without advanced mathematics. For instance, analytical geometry made possible CAD, advanced program, and these programs have contributed such as the fact that the new engine in the car occupies much less space than the old car models, calculation cutting surfaces etc. Also, computers and networks are based on advanced mathematics for the preservation of records, coding information etc. We all use mathematical perfect results without which on-line banking, WebTV, the Internet, radio etc., would not be possible (Ang, 2006). 
Modern technology will have absolute effects (full impact) on how future generations of mathematicians will discover and publish their mathematical results, share ideas and learn mathematics. Technology makes possible new methodologies for teaching mathematics and also will change the way that students learn mathematics and apply it in other disciplines. But modern technology, with all its advantages, cannot, in any way, replace a good teacher. A good teacher, equipped with effective tools and strategies to dominate the technology, can become an excellent teacher.

As Shakespeare said (Bernhard, 2000) "there is nothing either good or bad, but thinking makes it so". In the case of the use of modern technology in teaching mathematics, this statement may be paraphrased: "calculators and computers are not working means neither good nor bad-just using them makes them such". While driving the car, the most important is the driver-car is secondary. Also, the use of modern technology in teaching, first-hand role, plays the teacher, technology is second-hand.

\section{The Role of Technology in Mathematical Research}

Technology needs and requirements affect the presentation of new problems for researchers in mathematics. Although this effect is much smaller than the impact that technology has on science and other fields, however, it is important.

However, mathematics has some internal coherence, which allows it to develop its problems and to solve them without any reference to the outside world. Among other sciences, mathematics is the only one that owns this technique-no need to experiment. Despite the fact that this isolation is possible, it doesn't mean that this happens often, which means that this isolation is not necessary or useful.

Although mathematics can handle its problems in a higher degree than other sciences can, there are a large number of mathematical disciplines that have been developed later on, based on their beauty and their interesting character. It is sufficient to mention the example of geometry. It started as a practical tool for surveyors, however, when its importance was discovered, it began to develop as a separate branch. After a multi-year development, other branches have been developed, some of which are formed due to their secret beauty - which later have been applied-the others have been proposed by users. For example, differential geometry has been established from the famous Gauss to meet the needs of topography, while the Riemann geometry, unexpectedly, found application in the theory of relativity.

We could mention many other branches of mathematics, such as analysis, partial derivatives equations, Fourier analysis, etc.

Certainly there are areas that their application has not yet been recognized or their implementation has started too late. Among the well known in this regard is the number theory, which until recently has been completely not implemented in practice.

Algorithm, linear programming, game theory etc., are all disciplines that arose and developed as a necessity of solving many practical problems of practical reality of today.

It should be noted that not all that we want to do is valid. Sometimes, understanding of mathematics and the possibility of its implementation are the key challenges for the advancement of mathematics itself.

\section{Spiral Interaction between Technology and Mathematical Education}

Nowadays, even theoretical mathematicians admit that, with the use of LaTex, e-mail, Web-Surfing, econferences, etc. in the organization of scientific life, makes life of mathematicians much easier, and, in this way, we can see that global cooperation with colleagues is growing in terms of our results, as well as in research publications. This type of impact of computer technology on mathematics is not much different from the impact of this technology in other fields of science and engineering.

The existence of mathematical software, such as Mathematica, Maple, Derivative, Mat lab etc. in the past three decades, it has started to change the way that math is explained. Mathematical software has made great progress, both in advanced mathematics as well as in the simplest. In short, substantially all of the mathematics taught in secondary schools and in universities and engineering curricula, are already available on any computer. Everyday more teachers of mathematics are starting to use these systems to reorganize the learning of mathematics, but still, most of the curricula of mathematics are not using the advantages of the new technology, which is available.

Special emphasis should be given to the level of interaction between mathematical research and computer technology, which can be considered as interaction of fundamental importance, natural and inspiring. But we cannot leave without mentioning the fact that this interaction also, unfortunately, has not attracted enough attention of the mathematical community.

The relationship between computer technology and mathematical research is not expressed only as a twoway interaction, but can also be understood as an open spiral, where in each district of the spiral, known technology is used and at the same time when new technology is created.

\section{Preparing Teachers to Explain Mathematics Technology}

Almost all universities in the US and Europe are developing ways of providing courses (various materials) through the web. This will change the way of teaching mathematics and soon a small number of 
highly sophisticated virtual universities will attract a large number of students. In this way, basic education students will be offered a bargain price. This will be more helpful to students who are unable to attend regular class hours for work purposes or for any other reason. It will also create opportunities to study at a lower cost. From this aspect, technology will be a balancing mean, which will provide educational opportunities to people who, in other circumstances, would not have been able to study.

To find an appropriate way to use technology in education is also a major challenge not only for professors of mathematics but also for others. It requires effort and initial results may be in many cases perhaps even disappointing. But here we are all students who need to learn new things.

The appearance of technology at all levels of education has prompted the need to change teaching practices. Along with the development/modernization of optional learning projects for engineering and scientific subjects, it is important to focus on the preparation of future teachers of mathematics.

The use of technology in teaching mathematics activities may be regarded as a new language of communication in the development of construction of knowledge. Knowledge about the role of technology in the educational process can contribute to better preparation of future teachers in the right direction not only for technology but also the way of teaching.

Also, one of the advantages of technology as a work tool of teaching is also the possibility of resolving problems through modelling and interdisciplinary activities, so that new disciplines arise and are reformulated for the preparation of teachers of mathematics. Given this aspect, we can say that the interpretation of information that is given by the computer/calculator asks for the type of mathematical knowledge needed to use the technology. Careful use of technology as a teaching strategy improves lectures and preparation of activities by teachers.

About the importance of technology related to the professional development of teaching, Oldknow (2000) said, "The effective use of personal computer technology in supporting the mathematics curriculum is in the hands of teachers. They need to know more about the use of technology than can just be found from manuals, teaching materials and other information sources." (Oldknow, 2000).

Cornu also provides evaluation "Mathematics is evolving and changing under the influence of computer and informatics. Therefore, teachers need to maintain their mathematics knowledge and to practice mathematics from an informatics viewpoint. Mathematics is becoming more experimental, more algorithmic, more numerical; teachers must be able to follow the evolution of mathematics, and to acquire new competencies and new attitudes and to be able to carry out new activities in mathematics." (Waits \& Demana, 2000)

Therefore, some questions were submitted naturally as: "What is teaching through technology? What could change if technology is used in teaching? What are the ways of using technology in teaching and which of those are the most effective to achieve the educational objectives? "

One of the major challenges faced by teachers of mathematics when trying to introduce students to technology is that they do not know when, why and how to use it, even if they have prior knowledge regarding educational equipment and software. It also faces the challenge of university-level teachers, who have the responsibility to prepare future teachers with mathematical and pedagogical knowledge, required in modern teaching.

\section{Computer Use in Teaching Engineering Mathematics}

Using the computer as a tool to support teaching has been used a several years ago even in universities, especially in practical subjects. However, we should pose the question: are computers being used in teaching mathematics? The answer is obvious. In most cases they are used as calculators, since they are used as tools for calculating the numbers, although they are also used to algebraic operations, presenting curves etc.

This kind of use complements or simplifies traditional teaching methods, but this does not represent a significant improvement in the teaching of mathematics.

This is partly because teachers have been educated in an educational system, which did not have any training that had to do with technology. Thus, teachers do not have sufficient computer culture to prepare lectures to teach students adequately for their professional future. However significant improvement has been achieved: until a few years ago high school students have learned how to use a logarithmic tables. Now this is not necessary and is no longer taught; rather than that they use the computer or calculator. Without a doubt computer usage this calculation is faster, but let us be clear that this does not have improved the way of explaining the concept and use of log. In this way mathematicians have contributed with their work in creating the technological tools that are used by professionals of other professions relevant to their work.

In short, the challenge we have to face in the future is overcoming this situation in order to use the computer as a tool for the establishment of mathematical creativity. Since this is not an easy job, it is advisable that at least computer exercises are included in each branch where mathematics is used and, in particular, in engineering faculties. Thus, it will be possible for future professionals to be able to develop programs that will substantially change the way of explaining math.

The fact that students can perform these types of exercises with mathematical software's will help them not only in mathematics, but also in other courses. Also, they will be ready to face professional problems that they will face in the future. 


\section{Presenting Experiments}

It is broadly accepted that mathematical software's can contribute significantly to the learning and understanding of mathematics. Especially through visual possibilities of the software packages and computer algebra systems, students can investigate the behaviour of functions and phenomena that would be impossible without the help of computers. The teacher has a wide possibility of choices of software tools that they can use during their studies.

Several recent technological developments may have a very significant role in the study of functions. Especially important are graphic calculators and computers with appropriate software such as spreadsheets, graph plotters, and symbol manipulation programs. These technological instruments, wisely used in the mathematics classroom, may help students to develop a deeper sort of mathematical understanding, facilitating the process of conjecturing, and testing and making generalizations. This technology may also give students the necessary power to solve more difficult problems and suggest multiple links among such diverse domains as geometry, algebra, statistics, and real situations and their corresponding mathematical models.

However it still remains as a problem how to develop problems or interesting experiments, which will challenge students where technology is an important tool for investigating the problem, so that it allows the student to reflect reason, analyse, and modify their ideas until reaching a proper conclusion. A characteristic of these experiments is that without technology it would be difficult for students to do tests and reach the result, as the technology and its visual opportunities offer students mechanisms for experimentation and testing, allowing them to modify their hypotheses and their idea that leads them to a solution.

By exploring examples which work and examples, which fail, it is possible for the students to gain the visual intuitions necessary to provide powerful formal insights. Thus intuition and rigour need not be at odds with each other. By providing a suitably powerful context, intuition naturally leads into the rigour of mathematical proof. This is a nice role of technology that improves greatly the didactical transposition of mathematical concepts.

Example 1: The purpose of this experiment is to help students understand the concept of derivative related to the slope of tangent lines to the graphic of function in the given point $x=x_{1}$.

In this case, students are required to use computer software, to present graphic function $y=x^{2}$ and to examine what happens when $x=1$. Also, they are required to concentrate and analyse the point $(1,1)$ through enlarging (by zooming) graph of the function in that part. The idea that the function is differentiated in a point if it is locally straight is an important idea, which is much easier to understand than the concept of limit. Students can actually notice that if they enlarge graph of the function at any point the function will be locally straight across the area of definition. The more we zoom in, the more the parabola looks like a line. In other words the curve becomes almost indistinguishable from its tangent line. Figure 1 illustrates this procedure for the curve $y=x^{2}$. (James, 2008).
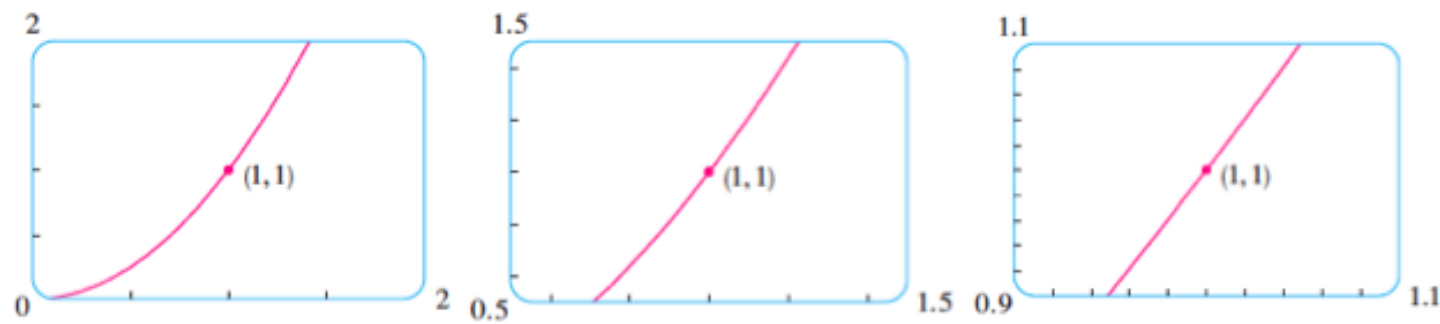

Figure-1. Zooming in toward the point $(1,1)$ on the parabola $y=x^{2}$.

Source: James Stewart “Calculus Early Transcendental Sixth Edition Mc Master University, 2008.

However, if students are asked to submit graphic of function $y=x|\sin x|$, they will be able to note that at the point $(0,0)$ the function is locally straight and to the point $(, 0)$ function is not locally straight, and thus not differentiated at that point. This can occur through animation, which helps the student to reinforce even more geometric concept of derivative.

It is clear that students, who have worked with these experiments, have developed a strong sense of geometric interpretation with respect to the derivative.

It isn't surprising that computer algebra systems (CAS) excel at integration. That doesn't mean that integration by hand is an obsolete skill. We will see that a hand computation sometimes produces an indefinite integral in a form that is more convenient than a machine answer. To begin, let's see what happens when we ask a machine to integrate the relatively simple function $y=x\left(x^{2}+5\right)^{8}$. 
Example 2: Use a CAS to evaluate $x\left(x^{2}+5\right)^{8} d x$.

Solution: Maple and Mathematica give the same answer:

$$
x\left(x^{2}+5\right)^{8} d x=\frac{1}{18} x^{18}+\frac{5}{2} x^{16}+50 x^{14}+\frac{1750}{3} x^{12}+4375 x^{10}+21785 x^{8}+\frac{218750}{3} x^{6}+156250 x^{4}+\frac{390625}{2} x^{2}
$$

It's clear that both systems must have expanded $\left(x^{2}+5\right)^{8}$ by the Binomial Theorem and then integrated each term.

If we integrate by hand instead, using the substitution $u=x^{2}+5$ we get

$$
x\left(x^{2}+5\right)^{8} d x=\frac{1}{18}\left(x^{2}+5\right)^{9}+c
$$

For most purposes, this is a more convenient form of the answer.

One of great features of technology in teaching mathematics is the graphing capabilities which enhances the understanding and development of mathematical thinking through visualization, possibilities of simulation, animation of models, etc.

It's very difficult to visualize a function $f$ of three variables by its graph, since that would lie in a fourdimensional space. However, we do gain some insight into $f$ by examining its level surfaces, which are the surfaces with equations $f(x, y, z)=k$, where $k$ is a constant. If the $(x, y, z)$ point moves along a level surface, the value of $f(x, y, z)$ remains fixed.

Example 3: Figure 2 shows some computer-generated level curves of the function

$$
f(x, y, z)=x^{2}+y^{2}+z^{2}
$$

Solution: The level surfaces are $x^{2}+y^{2}+z^{2}=k$, where $k \quad 0$. These form a family of concentric spheres with radius $\sqrt{k}$. Thus, as $(x, y, z)$ varies over any sphere with centre $\mathrm{O}$, the value of $f(x, y, z)$ remains fixed (James, 2008).

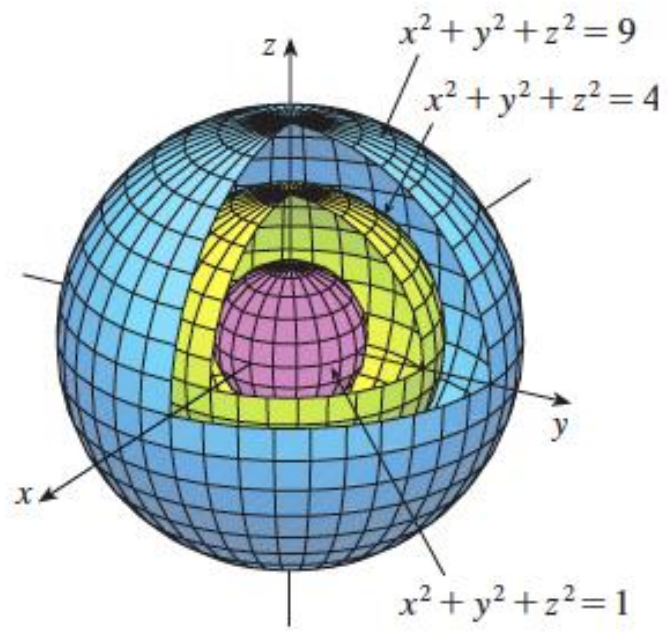

Figure-2. Family of concentric spheres.

Source: James Stewart "Calculus Early Transcendental Sixth Edition Mc Master University, 2008.

\section{Conclusion}

The new technology will provide tools that allow mathematicians to experiment with formulations and results, which enables them to reach deeper knowledge. The aim of mathematics is the movement of activity "pencil, paper and eraser" activity, in which the basic element is an advanced mathematical system such as Maple, Mat lab, Mathematica, Microsoft Mathematics, etc. This change is slow and gradual.

So we can conclude that: once advanced mathematics has changed the technology, and now advanced technology changes math! 
All that has already been said in this paragraph can be summarized with this saying: The community of mathematicians is building bridges that link the various mathematical disciplines, and some of these bridges are open for communication. A large number of them will not be possible without the use of new technology (Ang, 2006).

\section{References}

Ang, K. C. (2006). Mathematical modelling, technology and H3 mathematics. Mathematics Educator, 9(2), 3-47.

Bernhard, K. (2000). The algebraic calculator as a pedagogical tool for teaching mathematic. International Journal of Computer Algebra in Mathematics Education, 7(1), 5-23.

James, S. (2008). (6th ed.). Calculus early transcendental: Mc Master University.

Oldknow, A. (2000). Personal computing technology - use and possibilities, in hand-held technologies in mathematics and science education: A collection of papers. Laughbaum: The Ohio State University.

Waits, B., \& Demana, F. (2000). Calculators in mathematics teaching and learning: Past, present, and future. In Hand -held Technologies in Mathematics and Science Education: A Collection of Papers. Laughbaum: The Ohio State University. 